\title{
Bridging the Missing Link with Emicizumab: A Bispecific Antibody for Treatment of Hemophilia A
}

\author{
Georg Gelbenegger ${ }^{1}$ Christian Schoergenhofer ${ }^{1}$ Paul Knoebl ${ }^{2}$ Bernd jilma ${ }^{1}$ \\ ${ }^{1}$ Department of Clinical Pharmacology, Medical University of Vienna, \\ Vienna, Austria \\ 2 Division of Hematology, Department of Internal Medicine I, Medical \\ Address for correspondence Bernd jilma, MD, Department of Clinical \\ Pharmacology, Medical University of Vienna, Währinger Gürtel 18-20, \\ University of Vienna, Vienna, Austria
}

Thromb Haemost 2020;120:1357-1370.

\begin{abstract}
Hemophilia A, characterized by absent or ineffective coagulation factor VIII (FVIII), is a serious bleeding disorder that entails severe and potentially life-threatening bleeding events. Current standard therapy still involves replacement of FVIII, but is often complicated by the occurrence of neutralizing alloantibodies (inhibitors). Management of patients with inhibitors is challenging and necessitates immune tolerance induction for inhibitor eradication and the use of bypassing agents (activated prothrombin complex concentrates or recombinant activated factor VII), which are expensive and not always effective. Emicizumab is the first humanized bispecific monoclonal therapeutic antibody designed to replace the hemostatic function of activated FVIII by bridging activated factor IX and factor X (FX) to activate FX and allow the coagulation cascade to continue. In the majority of hemophilic patients with and without inhibitors, emicizumab reduced the annualized bleeding rate to almost zero in several clinical trials and demonstrated a good safety profile. However, the concurrent use of

Keywords

- hemophilia

- emicizumab

- bleeding

- bispecific antibody

- coagulation emicizumab and activated prothrombin complex concentrate imposes a high risk of thrombotic microangiopathy and thromboembolic events on patients and should be avoided. Yet, the management of breakthrough bleeds and surgery remains challenging with only limited evidence-based recommendations being available. This review summarizes published clinical trials and preliminary reports of emicizumab and discusses the clinical implications of emicizumab in treatment of hemophilia $\mathrm{A}$.
\end{abstract}

\section{Introduction}

Deficiency of coagulation factor VIII(FVIII), commonly known as hemophilia $\mathrm{A}$, is a severe bleeding disorder, ${ }^{1}$ representing 80 to $85 \%$ of the total hemophilia population. ${ }^{2}$ Hemophilia $\mathrm{A}$ is an $\mathrm{X}$-linked recessive bleeding disorder, thereby primarily affecting males. ${ }^{3}$ Hemophilia A is classified based on the residual FVIII activity level and is defined as severe (coagulation factor activity level $<1 \%$ ), moderate $(1-5 \%)$, or mild $(6-40 \%){ }^{4}$ The residual FVIII level depends on the type of mutation in the FVIII gene. Classification of hemophilia also correlates well with clinical profiles and bleeding symptoms. ${ }^{4,5}$ Approximately twothirds of patients with hemophilia A suffer from severe FVIII deficiency. ${ }^{6}$ Predominantly in patients with severe hemophilia A, total deficiency of FVIII can lead to serious joint bleedings, muscle bleedings, soft tissue bleedings, and life-threatening bleeding manifestations such as intracranial hemorrhages, 7,8 which can occur spontaneously. ${ }^{6}$ Hemophilic arthropathy is a serious complication of hemophilia-induced joint bleedings, ${ }^{9}$ caused by synovial inflammation with subsequent release of inflammatory cytokines and matrix-metalloproteases leading to progressive degradation of the cartilage. ${ }^{10}$ received

February 23, 2020

accepted after revision June 8,2020
DOI https://doi.org/

10.1055/s-0040-1714279.

ISSN 0340-6245. (c) 2020 Georg Thieme Verlag KG Stuttgart · New York
License terms

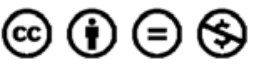




\section{A physiologic hemostasis}

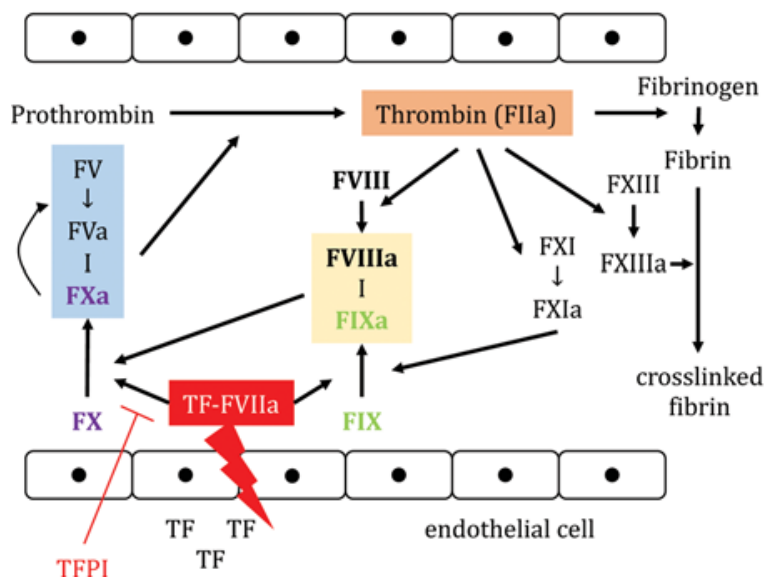

B hemophilia A treated with emicizumab

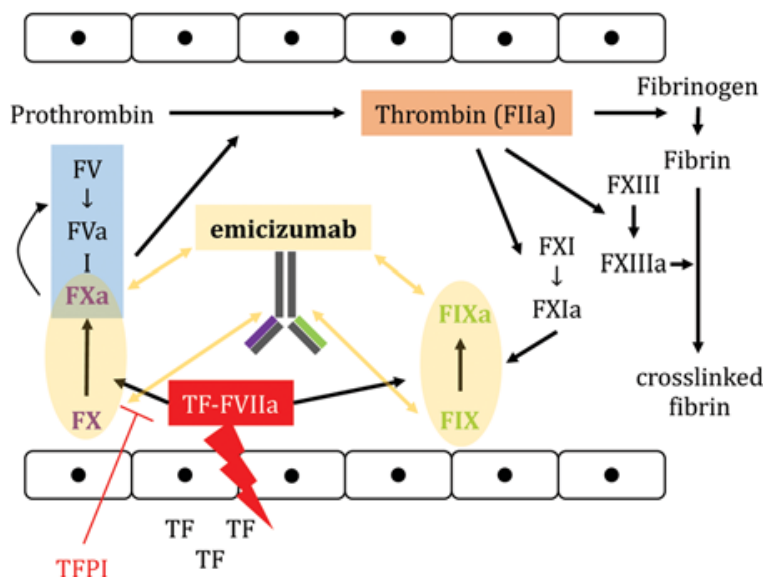

Fig. 1 Schematic comparison of (A) physiologic hemostasis and (B) hemostasis in hemophilia A under treatment with emicizumab.

\section{Role of Factor VIII in the Coagulation Cascade}

It is well recognized that the intrinsic pathway is not an accurate model of hemostasis in vivo. ${ }^{11}$ Activation of the coagulation cascade is mostly triggered by the tissue factor (TF) pathway, which involves complex formation of TF and activated factor VII (FVIIa) at the site of injury (-Fig. 1A). The TF/FVIIa complex not only activates factor X (FX) directly, but also activates factor XI (FIX) which further sustains activated FX (FXa), leading to generation of thrombin. Thrombin itself may also activate FXI and FVIII, thereby creating a positive feedback loop. ${ }^{12}$ FXa when associated with phospholipids or the TF/FVIIa complex is also a potent activator of FVIII. ${ }^{13,14}$ Although not causally involved in the activation process of coagulation, FVIII helps to further maintain and strengthen the production of FXa, ultimately resulting in the generation of thrombin and formation of a stable fibrin clot. In patients with hemophilia A, lack or dysfunction of FVIII impedes physiologic coagulation function and increases the risk of bleeding. $^{15}$

\section{Factor Replacement Therapy}

FVIII replacement with FVIII concentrates is still the treatment of choice in patients suffering from moderate and, in particular, severe hemophilia. ${ }^{2,16}$ FVIII concentrates can either be plasma-derived (originating from human donor blood) or recombinant (biotechnologically produced from genetically modified cells). Several modified recombinant FVIII products with extended half-lives have been released to increase dosing intervals and obtain higher trough levels. ${ }^{17}$ Treatment can either be prophylactic or episodic. Prophylactic FVIII replacement has been shown to significantly reduce bleeding events ${ }^{18-22}$ and slow down joint deterioration. ${ }^{23}$ While prophylactic treatment is the more effective way to prevent bleeding and long-term complications, ${ }^{18}$ it is expensive, burdensome, and may rarely necessitate central venous access which is associated with infection and thrombosis. Alternatively, episodic FVIII replacement therapy may be suitable for patients with mild or moderate factor deficiency and a milder bleeding phenotype. The decision which patient population is eligible for and should receive episodic or prophylactic replacement therapy depends on individual patient-related factors (rate and severity of bleeding, venous access, personal preference, etc.). ${ }^{2}$

Yet, there are considerable downsides to FVIII replacement. First, it is expensive and not available everywhere. So far, FVIII can only be given intravenously, implicating that repeated venipunctures are required for a regular, long-term treatment. This often coincides with a reduced patient compliance followed by a worse outcome. ${ }^{24}$ The use of FVIII replacement therapy is further complicated by a high interindividual variability in FVIII pharmacokinetics (PK), which necessitates tailored treatment regimens for individuals leading to frequent dose corrections to maintain sufficient FVIII trough levels. ${ }^{25}$ In the past, FVIII replacement using plasma-derived concentrates has led to the transmission of blood-borne diseases including hepatitis B and C, and human immunodeficiency virus, ${ }^{26-30}$ which has caused significant morbidity and mortality. Since then, improvement in processing of FVIII concentrates has led to a significant decrease of viral infections; however, pathogen transmission through plasma-derived FVIII concentrate infusion yet remains a potential risk.

\section{Inhibitors}

Probably the most challenging aspect of factor replacement therapy in patients with hemophilia $A$ is the occurrence of inhibitory alloantibodies against FVIII. Such inhibitors can rise to very high titers (high responders) and neutralize circulating FVIII rendering replacement therapy ineffective. In patients with severe hemophilia $\mathrm{A}$, anti-FVIII antibodies form in 25 to $40 \%$ within the first 50 exposure days. ${ }^{24}$ FVIII inhibitors are associated with increased mortality, ${ }^{31}$ limitations of physical function, ${ }^{32}$ orthopedic complications, ${ }^{33}$ and decreased quality of life $^{34}$ and increased health care costs when compared with patients without inhibitors. ${ }^{35,36}$ Inhibitors necessitate alternative treatment approaches such as 
immune tolerance induction (ITI) or the use of bypassing agents (BPAs), recombinant FVIIa (rFVIIa, NovoSeven), and activated prothrombin complex concentrate (APCC, FEIBA). ${ }^{37,38}$ However, the use of BPAs is not as effective as replacement of FVIII, ${ }^{38-40}$ and has several other disadvantages (very high costs, short half-life, and risk of thromboembolic events). Despite its mechanism of inhibitor elimination still being unclear, ITI shows a response rate of 50 to $75 \%,{ }^{41,42}$ yet, it requires long-term infusion of high doses of FVIII concentrates and can take years to show effect. ${ }^{43}$ Treatment of hemophilia A complicated by FVIII inhibitors requires a high amount of human and economic resources. ${ }^{44}$

In recent years, multiple novel FVIII products have been made available, mostly improving PK aspects, but showing varying results with respect to immunogenicity. ${ }^{7,45}$ However, development of FVIII inhibitors substantially limits the use of factor replacement therapy. Therefore, the unmet medical need for a treatment approach for hemophilia that is easy to use, with a long-lasting effect regardless of the presence of FVIII inhibitors, has led to the development of emicizumab, a new bispecific therapeutic antibody mimicking the effect of FVIII.

This review focuses on the safety and efficacy of emicizumab and discusses its clinical implications and potential extended use.

\section{Emicizumab}

Emicizumab (Hemlibra, Roche, Switzerland) is the first commercially available nonfactor replacement product for treatment of congenital hemophilia A. In the United States, it was first approved by the Food and Drug Administration (FDA) in 2017 for the use in patients with congenital hemophilia A with inhibitors, with its indication subsequently being extended for prophylactic use in hemophilic patients with and without inhibitors in 2018. In the European Union, emicizumab is approved for the routine prophylaxis of bleeding episodes in patients with congenital hemophilia A with FVIII inhibitors or severe hemophilia A without inhibitors. ${ }^{46}$

Emicizumab is injected subcutaneously with FDA-approved maintenance dose regimens of $1.5 \mathrm{mg} / \mathrm{kg}$ once every week (QW), $3 \mathrm{mg} / \mathrm{kg}$ once every 2 weeks (Q2W), or $6 \mathrm{mg} / \mathrm{kg}$ once every 4 weeks ( $4 \mathrm{~W})$. The recommended loading dose for all treatment regimens is $3 \mathrm{mg} / \mathrm{kg}$ QW for the first 4 weeks.

So far, results from four large HAVEN trials have been published (-Tables $\mathbf{1}$ and $\mathbf{2}$ ).

\section{Mechanism of Action and Pharmacodynamic Profile}

Emicizumab is a humanized bispecific monoclonal antibody (IgG4) that binds to both the activated FIX (FIXa) and FX. It is therefore designed to mimic the function of missing or deficient FVIII, which is an essential part of effective hemostasis. Emicizumab aligns FIXa and FX in a suitable spatial position, thereby promoting the interaction between the two coagulation factors and accelerating FX activation by $\mathrm{FIXa}^{47,48}$ ( - Fig. 1B). However, there are considerable differences be- tween the natural FVIII and emicizumab. As expected for an antibody, emicizumab binds only to a single site within the FIX and FX molecules, whereas FVIII has multiple interaction sites. Therefore, emicizumab shows substantially less affinity for its substrates, it cannot differentiate between zymogen and enzyme (FIX and FX vs. FIXa and FXa), and it lacks natural regulation of its activity, i.e., the activation by thrombin and the inactivation by protein $\mathrm{C}^{49}$ Due to its structural difference to FVIII, emicizumab does not induce the development of FVIII inhibitors. $^{50}$

The pharmacodynamic (PD) response to emicizumab correlates with plasma concentrations of emicizumab. In ex vivo FVIII-neutralized plasma from healthy subjects who received emicizumab in different doses, emicizumab dose-dependently shortened the activated partial thromboplastin time (aPTT). Similarly, peak thrombin generation increased in a dosedependent manner. In healthy volunteers without FVIII neutralization, emicizumab only slightly shortened the aPTT but did not increase peak thrombin generation. ${ }^{51}$ This minimal effect of emicizumab in healthy volunteers can be explained by the higher affinity of FVIIIa than that of emicizumab for FXa. ${ }^{49}$ In patients with hemophilia $\mathrm{A}$, emicizumab dose-dependently shortened the aPTT and increased peak thrombin generation. $^{52}$ Initiation of emicizumab $(6 \mathrm{mg} / \mathrm{kg})$ in hemophilic patients normalized the aPTT within 8 hours and increased peak thrombin generation over the following weeks. ${ }^{53}$

\section{Pharmacokinetic Profile and Immunogenicity}

Following subcutaneous injection of $1 \mathrm{mg} / \mathrm{kg}$, emicizumab showed an absolute bioavailability of 80 to $93 \%$ and a mean absorption half-life of 1.7 days. ${ }^{54}$ The mean elimination halflife of emicizumab is 1 month ranging from 28.8 to 34.4 days (-Table 3). ${ }^{51}$ The long half-life of emicizumab is assumed to be due to its IgG4 structure including an altered amino acid sequence that lowers its isoelectric point and reduces its elimination. ${ }^{54}$ The mean apparent volume of distribution of emicizumab is $11.4 \mathrm{~L}$. Plasma concentrations of emicizumab increased in a dose-dependent manner. ${ }^{51,52}$ Emicizumab showed a first-order elimination phase. ${ }^{51}$ Mean trough plasma concentrations depend on the applied dose regimen (after a loading dose of $3 \mathrm{mg} / \mathrm{kg}$ QW for 4 weeks): when emicizumab was given $\mathrm{QW}$ at a dose of $1.5 \mathrm{mg} / \mathrm{kg}$, mean trough plasma levels were above $50 \mu \mathrm{l} / \mathrm{mL}^{55-57}$ Dosing regimens injecting emicizumab Q2W $(3 \mathrm{mg} / \mathrm{kg})$ and Q4W $(6$ $\mathrm{mg} / \mathrm{kg}$ ) resulted in mean trough plasma levels of 45 to $50^{55,57}$ and 38 to $40 \mu \mathrm{g} / \mathrm{mL}, 53,57$ respectively (-Table 3). The PK of emicizumab are unaffected by age (1-77 years), race, inhibitor status, mild or moderate hepatic impairment, and mild or moderate renal impairment. ${ }^{46,58}$

Further, the immunogenic potential of emicizumab remains to be better defined. Six studies provide data on the occurrence of antidrug antibodies in healthy subjects or patients undergoing treatment with emicizumab ( - Table 3). ${ }^{51-53,55-57}$ In the first-in-man trial, one subject tested positive for anti-emicizumab antibodies even at baseline (before drug administration) and another developed de novo antibodies causing a shortening 


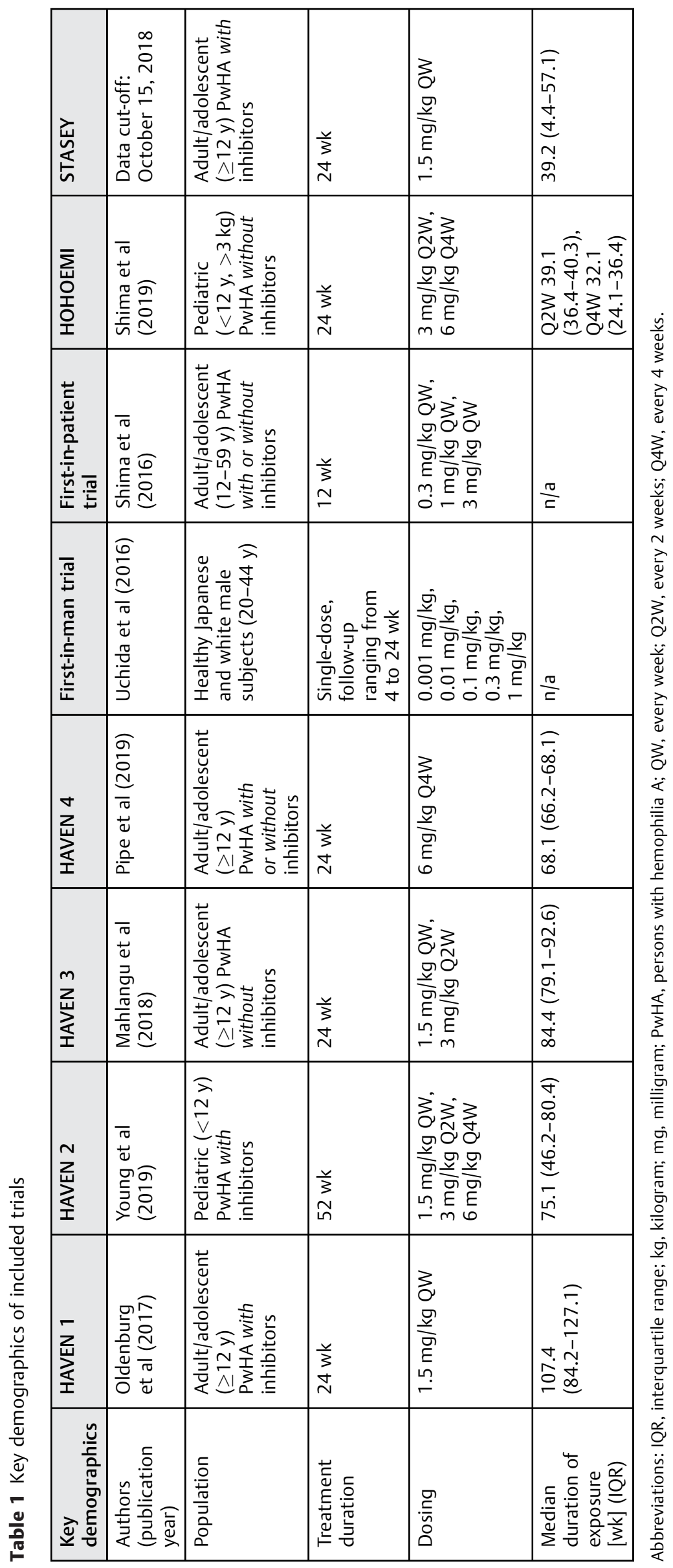

Thrombosis and Haemostasis Vol. 120 No. 10/2020 


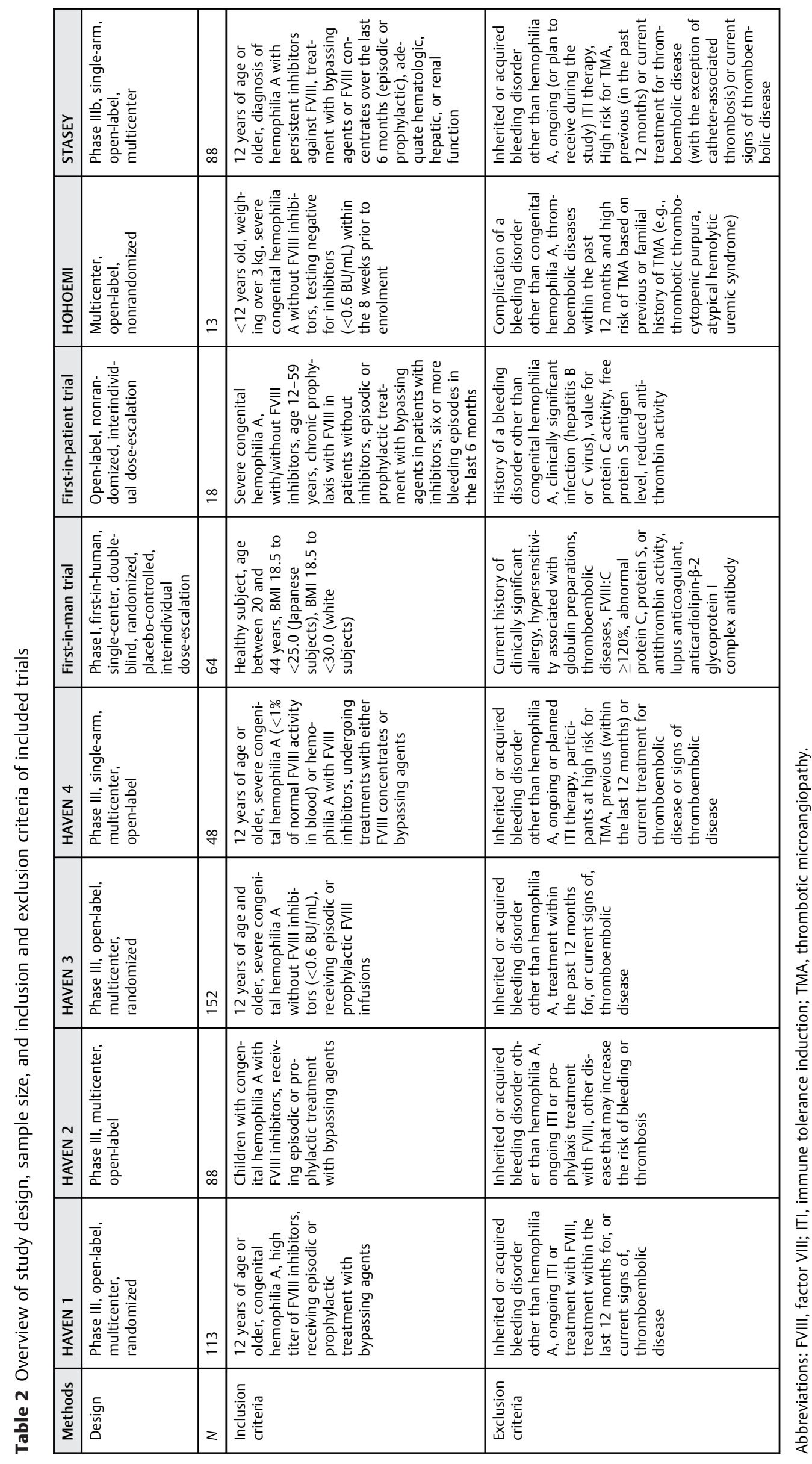




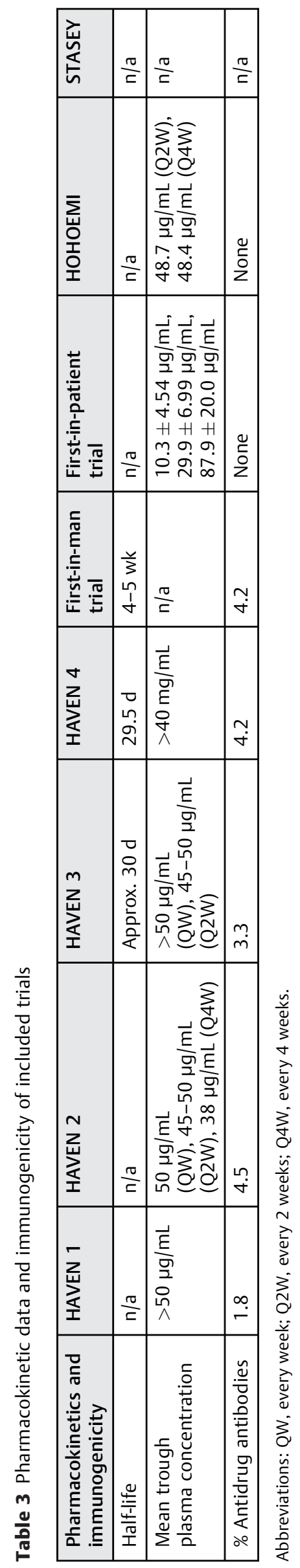

of emicizumab half-life. ${ }^{51}$ The first study of emicizumab in hemophilic patients found antidrug antibodies in one patient at baseline, but without neutralizing ability. ${ }^{52}$ In the HAVEN 1 trial, no subject tested positive for antidrug antibodies; however, two patients showed PK profiles indicative of such. ${ }^{56}$ The HAVEN 2 trial identified four patients with antidrug antibodies, two of which had neutralizing ability. ${ }^{57}$ In HAVEN 3, no de novo antidrug antibodies were identified; however, measurement discrepancies occurred in two patients and one patient intermittently tested positive after ITI. ${ }^{55}$ No antidrug antibodies were found in patients in HAVEN $4 .{ }^{53}$ Due to the limited sample size of studies available, it is difficult to accurately derive the incidence of antidrug antibodies and the proportion of antibodies with neutralizing potential. Further studies with longterm follow-up periods are needed to better characterize immunogenicity.

\section{Efficacy}

\section{Overall Analysis}

Pooled data from HAVEN 1-4 revealed the annualized bleeding rate $(A B R)$ under treatment with emicizumab to be 1.5 (95\% confidence interval [CI]: $1.20-1.84$ ) over a median duration of exposure of 83 weeks. ${ }^{59}$ Three different treatment regimens have been tested in the HAVEN trials (-Table 4): subcutaneous injection of emicizumab of 1.5 $\mathrm{mg} / \mathrm{kg}$ QW, $3 \mathrm{mg} / \mathrm{kg} \mathrm{Q} 2 \mathrm{~W}$, or $6 \mathrm{mg} / \mathrm{kg}$ Q4W. All treatment regimens were preceded by four loading doses of $3 \mathrm{mg} / \mathrm{kg}$ QW. Emicizumab QW, Q2W, and Q4W regimens were associated with a mean ABR across HAVEN 1-4 of 1.6, 0.8, and 2.3, respectively. ABRs of the four individual HAVEN trials are shown in - Table 4. In every treatment cohort of the HAVEN trials, at least $50 \%$ of patients (range $56-90 \%$ ) had zero treated bleeding events. The proportion of patients with no bleeding events increased over time in all four trials. Across HAVEN 1-4, emicizumab was associated with an ABR for spontaneous bleeds of 0.6 (95\% CI: $0.5-0.8)$ and for joint bleeds of 1.0 (95\% CI: 0.8-1.3). Again, the proportion of patients with zero bleeding events (spontaneous and joint) increased over time. Further, emicizumab was associated with a resolution of $99 \%$ of target joints (target joints were defined as major joints [e.g., hip, elbow, wrist, shoulder, knee, and ankle] in which $\geq 3$ bleeding events occurred over a 24-week period; target joint resolution was defined as $\leq 2$ bleeding events in a 52-week period in a joint previously defined as a target joint) in patients across HAVEN 1-4. ${ }^{59}$

In the Japanese pediatric HOHOEMI trial, although small in sample size, Q2W and Q4W treatment regimens showed ABRs for treated bleeding events of 1.3 and 0.7 , respectively. ${ }^{60}$ Similar to previous studies, interim results from the STASEY trial report an ABR of 0.5 for treated bleeding events. ${ }^{61}$

\section{Comparison of Efficacy in Patients with versus without FVIII Inhibitors}

Three studies, HAVEN 1, 2, and STASEY, evaluated the efficacy of emicizumab in hemophilic patients with FVIII inhibitors only. ${ }^{56,57}$ Emicizumab resulted in ABRs of treated bleeds of 2.9, 0.3 , and 0.5 (all QW), respectively. 


\begin{tabular}{|c|c|c|c|c|c|c|c|c|}
\hline 岀 & 文 & 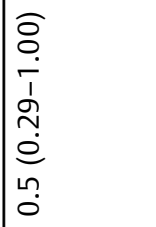 & $\hat{\dot{\infty}}$ & 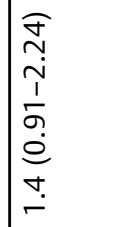 & $\mid \begin{array}{c}\widetilde{f} \\
\tilde{n} \\
0 \\
1 \\
0 \\
0 \\
0 \\
0 \\
n \\
0\end{array}$ & $\mid \begin{array}{c}0 \\
0 \\
0 \\
0 \\
1 \\
0 \\
0 \\
0 \\
m \\
0 \\
0\end{array}$ & $\mid \begin{array}{c}0 \\
0 \\
0 \\
1 \\
1 \\
0 \\
0 \\
0 \\
\vdots \\
0\end{array}$ & $\frac{\pi}{2}$ \\
\hline $\begin{array}{l}\sum_{\text {I }} \\
\text { 꼰 } \\
\text { 오 }\end{array}$ & 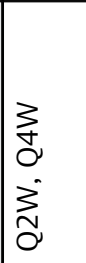 & 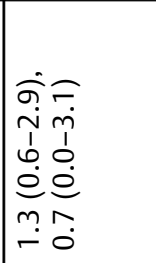 & $\begin{array}{l}\vec{\nabla} \\
\stackrel{i}{*} \\
\dot{m} \\
m \\
m\end{array}$ & 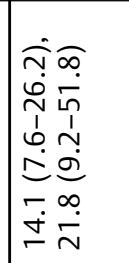 & 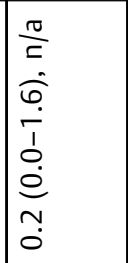 & 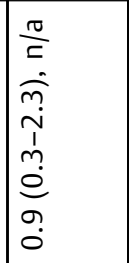 & $\begin{array}{l}\frac{\pi}{a} \\
\frac{\pi}{a}\end{array}$ & $\begin{array}{l}\frac{\pi}{L} \\
\frac{\pi}{L}\end{array}$ \\
\hline 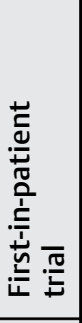 & 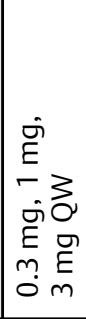 & $\frac{\pi}{a}$ & 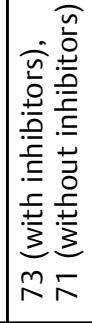 & 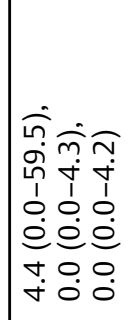 & $\frac{\pi}{x}$ & $\frac{\pi}{\Omega}$ & $\frac{\pi}{\Sigma}$ & $\frac{\pi}{2}$ \\
\hline 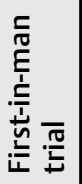 & $\frac{\pi}{2}$ & $\frac{\pi}{2}$ & $\frac{\pi}{2}$ & $\frac{\pi}{2}$ & $\frac{\pi}{2}$ & $\frac{\pi}{2}$ & $\frac{\pi}{2}$ & $\frac{\pi}{2}$ \\
\hline 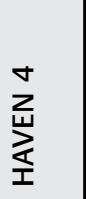 & $\underset{0}{3}$ & 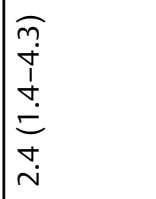 & $\overline{\dot{\varphi}}$ & 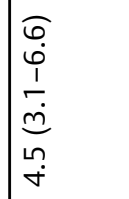 & 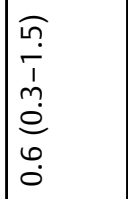 & 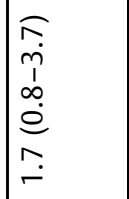 & 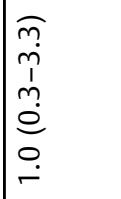 & $\stackrel{\circ}{\circ}$ \\
\hline 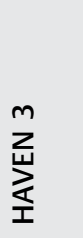 & 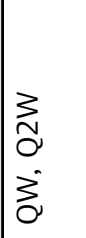 & 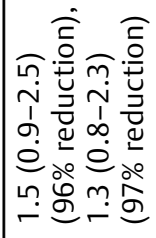 & $\begin{array}{l}0 \\
0 \\
0 \\
0 \\
0 \\
\text { ผ่. } \\
\text { ผn }\end{array}$ & 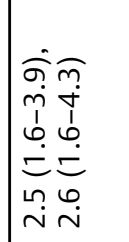 & 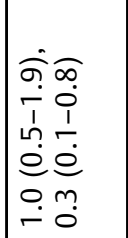 & 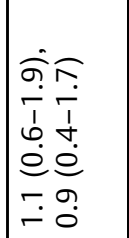 & 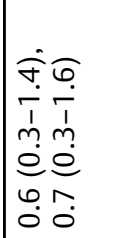 & مَ \\
\hline$\underset{\text { 妾 }}{\stackrel{N}{\geq}}$ & $\mid \begin{array}{l}3 \\
0 \\
0 \\
3 \\
\vdots \\
0 \\
3 \\
0\end{array}$ & 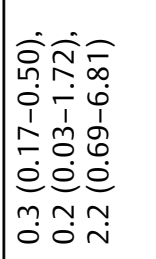 & $\begin{array}{l}0 \\
\dot{8} \\
0 \\
\dot{0} \\
\dot{0} \\
0 \\
0 \\
0\end{array}$ & 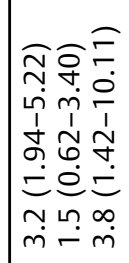 & 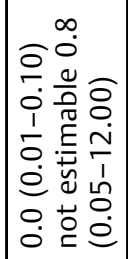 & 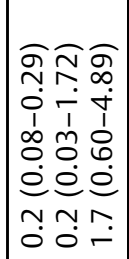 & 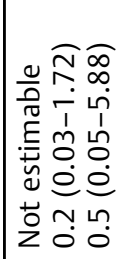 & $\stackrel{8}{\circ}$ \\
\hline 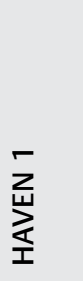 & 文 & 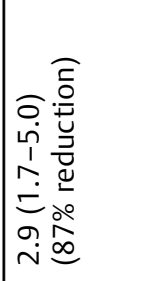 & $\hat{\theta}$ & $\begin{array}{l}0 \\
0 \\
0 \\
0 \\
1 \\
0 \\
0 \\
0 \\
n \\
n \\
n\end{array}$ & 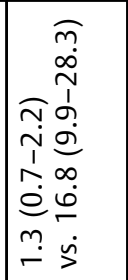 & 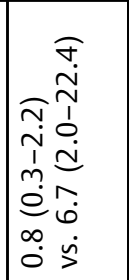 & $\begin{array}{ll} & \\
& \\
0 & 0 \\
0 & 1 \\
0 & 0 \\
1 & 0 \\
0 & 0 \\
0 & 0 \\
0 & 0 \\
- & m \\
0 & \dot{n}\end{array}$ & $\begin{array}{l}\hat{\infty} \\
\infty\end{array}$ \\
\hline 矛 & 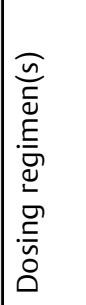 & 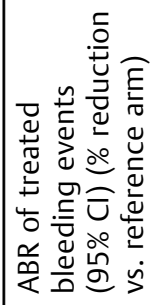 & 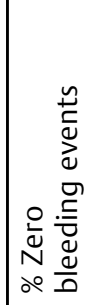 & 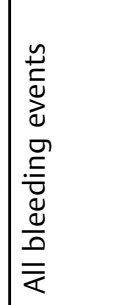 & 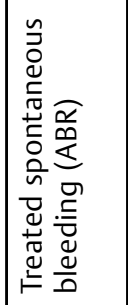 & 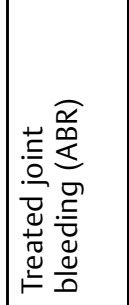 & 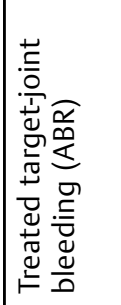 & 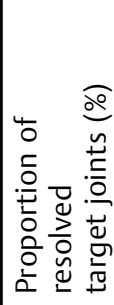 \\
\hline
\end{tabular}


Two studies, HAVEN 3 and HOHOEMI, only included patients without FVIII inhibitors. ${ }^{55,60}$ Emicizumab was associated with ABRs of treated bleeds of $1.5(\mathrm{QW})$ and 1.3 (Q2W), respectively. HAVEN 4 included both patients with and without inhibitors; however, $88 \%$ of patients in the expansion cohort had no FVIII inhibitors. The ABR for treated bleeds in HAVEN 4 was $2.4(\mathrm{Q} 4 \mathrm{~W})$.

\section{Comparison of Efficacy According to Different} Treatment Regimens ( $Q W$ versus $\mathbf{Q} \mathbf{W}$ versus $\mathbf{Q} 4 \mathrm{~W}$ )

Six studies evaluated three treatment regimens. The median ABR of treated bleeds of the QW, Q2W, and Q4W treatment regimens, pooled from all studies, was $1,1.3$, and 2.2, respectively.

\section{Safety and Drug Interactions}

Injection-site reactions were the most common treatmentrelated adverse events and frequently reported across all HAVEN trials $(n=104 ; 26.1 \%) .{ }^{59}$ All injection-site reactions were of mild or moderate severity, most of which (93\%) resolved without treatment. Other adverse events included headache (15\%), arthralgia (15\%), pyrexia (6\%), and diarrhea (6\%). ${ }^{58} \mathrm{~A}$ total of 103 serious adverse events were reported in 71 participants with hemorrhage and hemarthrosis being reported by $\geq 5$ participants.

Adverse events of special interest occurred in five patients of the HAVEN 1 trial. Of these, three patients developed thrombotic microangiopathy (TMA), one patient suffered from cavernous sinus thrombosis, and another patient suffered from skin necrosis/superficial thrombophlebitis. One patient with TMA developed rectal bleeding, refused treatment with blood transfusions, and subsequently died. His death was considered unrelated to emicizumab due to the resolving TMA at the time.

Notably, the occurrence of all five coagulation-associated adverse events was associated with the concomitant use of APCC at doses of $>100 \mathrm{U} / \mathrm{kg} / \mathrm{d}$ for a time period of more than 1 day. None of the patients receiving concomitant treatment with emicizumab and APCC of any dose for only 1 day had any thromboembolic events. Otherwise, in a retrospective analysis of real-world patients, one patient developed postoperative thrombosis/TMA in association with APCC despite emicizumab discontinuation 1 month prior to the surgery. ${ }^{62}$ The pathomechanism of TMA caused by concomitant use of emicizumab and APCC is probably the crosslinking of the activated factors contained in the APCC. ${ }^{54}$ Interestingly, the incidence of TMA was limited to APCC only; no cases of TMA or thromboembolic events have been linked to the concomitant use of rFVIIa. Moreover, management of bleeding episodes with FVIII in emicizumab-treated patients without inhibitors was also not associated with the occurrence of TMA or thromboembolic events.

One patient in the HAVEN 2 trial was believed to suffer from a systemic hypersensitivity reaction due to symptoms of abdominal pain and cough but upon later medical review his symptoms were confirmed not to be linked to anaphylaxis.
In the HOHOEMI trial, two patients experienced serious adverse events unrelated to the study drug. No thromboembolic events, TMA, or systemic hypersensitivity reactions were observed. $^{60}$

In the STASEY trial, of 17 patients who experienced a treated bleeding event, 16 were concomitantly treated with rFVIIa and one was treated with standard FVIII. There was no occurrence of any thromboembolic event or TMA. ${ }^{61}$

\section{Surgery}

In total, $31.6 \%(126 / 399)$ of patients in HAVEN 1-4 underwent at least one surgical procedure. ${ }^{63}$ of 233 surgeries performed, 18 were deemed major surgeries and 215 were deemed minor surgeries. One hundred forty-one of the minor surgeries were managed without prophylactic infusion of coagulation factor and over $90 \%$ of patients had no treated postoperative bleeding events. Of the 18 major surgeries, all three that were managed without prophylactic administration of coagulation factor had no treated postoperative bleeds. Of the remaining 15 patients treated with prophylactic coagulation factor, only one had a treated bleeding event. Major surgeries included five cases of arthroplasty with only one occurrence of bleeding due to surgery.

Further, the successful use of emicizumab with perioperative administrations of rFVIIa in a major surgery has been described in patients receiving total hip arthroplasty. ${ }^{64,65}$ Another patient with acquired hemophilia A under treatment with emicizumab underwent percutaneous coronary intervention and received periprocedural loading with $325 \mathrm{mg}$ aspirin and $600 \mathrm{mg}$ clopidogrel. He was successfully treated and continued dual-antiplatelet therapy without breakthrough bleeding events or need for BPA administration. ${ }^{66}$

\section{Health-Related Quality of Life}

Health-related quality of life (HR-QoL) was assessed using the Haem-A-QoL physical health subscale score at week 25. Generally, improvement of HR-QoL was seen across all published HAVEN trials. Further, emicizumab was associated with fewer missed days at school or work ${ }^{53,67}$ and a reduced number of days hospitalized. ${ }^{67}$ Almost all patients preferred emicizumab treatment over prophylaxis with FVIII or BPAs. ${ }^{53,55}$

\section{Laboratory Testing}

Assessment of the hemostatic function and FVIII activity in patients under treatment with emicizumab needs special attention. ${ }^{68,69}$ Since emicizumab causes a significant shortening of the aPTT even at very low concentrations, ${ }^{52,69}$ use of conventional aPTT tests is futile. Diluted aPTT, however, correlates well with the plasma emicizumab concentration. ${ }^{70}$

Conventional FVIII activity assays were found to be either oversensitive or insensitive to emicizumab, which precludes their use. However, a modified FVIII one-stage assay calibrated against emicizumab accurately quantifies FVIII activity and measures emicizumab concentrations. ${ }^{71}$ 
To differentiate between the PD effect of emicizumab and the endogenous residual FVIII activity, chromogenic FVIII assays using two different reagents need to be used. ${ }^{72}$ While the chromogenic assay using bovine reagents can detect the endogenous FVIII activity, the one using human reagents assesses emicizumab's effect. ${ }^{69}$

For measurement of inhibitors under concurrent treatment with emicizumab, a chromogenic Bethesda assay using bovine reagents can be used. ${ }^{73}$

Although not routinely done, rotational thromboelastometry (ROTEM; Pentapharm GmbH, Munich, Germany) may be used for assessment of coagulation function in emicizumabtreated patients. In particular, clotting time and clot formation time of the nonactivated thromboelastometry test (native coagulation, NATEM) showed a dose-dependent response to emicizumab. ${ }^{74}$ Thromboelastometry has also been used to assess coagulation function in an in vitro experiment involving emicizumab, APCC, and rFVIIa. ${ }^{75}$ Clot waveform analysis may also be a promising tool for coagulation assessment. ${ }^{76}$ The thrombin generation assay has been shown to be a useful marker of hemostatic function in patients under treatment with emicizumab ${ }^{77}$ and is also helpful in the assessment of coagulation in emicizumab-treated patients receiving concomitant treatment with BPAs. ${ }^{78}$

\section{Discussion}

So far, three large trials (HAVEN 1, 3, and 4), evaluating the safety, efficacy, and PK of emicizumab in adults and adolescents with hemophilia $A$, have been published. ${ }^{53,55,56}$ All three of them showed emicizumab to significantly reduce ABRs. Additionally, results from the HAVEN 2 trial, including only children $<12$ years ( $2-11$ years), have been published, further substantiating the findings of previous studies. ${ }^{57}$ The recently published HOHOEMI trial and interim results from the STASEY trial also confirmed emicizumab's efficacy. ${ }^{60,61}$

While showing a significant decrease in bleeding events, the HAVEN trials lack prospective data comparing emicizumab versus standard prophylaxis with either FVIII or BPAs. Instead, direct comparison of emicizumab with standard care is performed through a noninterventional study, comparing intraindividual patient data. Of note, HAVEN 4 features only a single experimental arm (Q4W).

Intraindividual comparison of HAVEN 3, including hemophilic patients without inhibitors, who previously received prophylaxis with FVIII, showed emicizumab prophylaxis to be associated with a $68 \%$ lower ABR.

Noninterventional study results from HAVEN 1 and 2, including hemophilic patients with inhibitors under previous prophylactic treatment with BPAs, showed a 79 and $99 \%$ decreased ABR, respectively, with emicizumab prophylaxis, indicating a larger effect size of emicizumab prophylaxis in inhibitor patients.

\section{Bleeding under Treatment with Emicizumab}

Regardless of the impressive clinical performance of emicizumab, breakthrough bleeding events may still occur and call for additional episodic treatment with either FVIII or BPAs, depending on the presence of inhibitors. Management of breakthrough bleeding proves especially challenging in patients with inhibitors as outlined by the HAVEN 1 trial.

While the concurrent use of APCC and emicizumab imposes a high prothrombotic risk, the concomitant use of rFVIIa in the context of breakthrough bleeding events under emicizumab prophylaxis has so far not been associated with TMA or thromboembolic events ${ }^{79}$ and is recommended as first-line treatment in a recent guidance paper by the United Kingdom Haemophilia Centre Doctors' Organisation (UKHCDO $){ }^{80}$ If, after administration of rFVIIa, the bleeding event does not resolve, treatment with FVIII should be initiated. Usage of APCC should only be considered unless no other option is available. ${ }^{80}$

Another review from the French network on inherited bleeding disorders (MHEMO), the French Reference Centre on Haemophilia, in collaboration with the French Working Group on Perioperative Haemostasis (GIHP) also favors rFVIIa as first-line treatment in a bleeding patient with inhibitors treated with emicizumab, with a similar downstream treatment algorithm. Optionally, tranexamic acid can be given as a supportive measure to rFVIIa. ${ }^{81}$

Further, the Italian Association of Haemophilia Centres (AICE) have provided a practical guidance paper on how to manage patients with hemophilia $A$ and inhibitors under treatment with emicizumab in the emergency department. ${ }^{82}$

However, the uncritical, permissive use of rFVIIa in emicizumab-treated patients is unwarranted, since the results of the bleeding analysis from HAVEN 1, 2, and 4 show some major limitations. ${ }^{79}$ First, universal application of the study is precluded by the exclusion of patients with a high thrombotic risk from the included trials. Second, around half of the patients were exposed to only a single-dose treatment of rFVIIa, leaving information on repeated use unknown. Lastly, the study lacks information on patients in the clinical setting of infection (sepsis), trauma, and major surgery. ${ }^{83}$

In hemophiliacs without inhibitors, however, breakthrough bleeding can easily be treated with FVIII concentrates, which should be administered as soon as possible. For joint bleeds and soft tissue bleeds, a dose of approximately 25 units/kg is recommended. ${ }^{84}$

\section{Emicizumab versus Standard-of-Care Treatment}

Emicizumab's exact place in future guidelines remains to be further discussed, yet, convincing arguments can be made for its primary use.

First, patients with poor venous access are spared repeated venipuncture attempts or even the installation of an intravenous catheter, which involves the risk of thrombosis and infection.

Second, in previously untreated patients (PUPs), the initiation of emicizumab could enable (almost) complete discontinuation of FVIII replacement, which includes the prevention of inhibitor development in the first place. In that case, FVIII concentrates would exceptionally be used to treat breakthrough bleeding events, which might potentially slow down or even preclude the development of inhibitors. Inhibitor development is not only dependent on the timing of FVIII 
infusion but also on the frequency and intensity of treatment (e.g., during bleeding episodes). ${ }^{85,86}$ During HAVEN 3, emicizumab reduced the ABR to zero in more than $50 \%$ of patients, thereby completely eliminating the need for FVIII coadministration in these patients. If, in the remaining patients, episodic administration of FVIII was required (in total 215 coexposure events with emicizumab and FVIII were reported in 64 patients), most doses of FVIII administered were very low ( $<50 \mathrm{IU} / \mathrm{kg}$ bodyweight) and given over a time frame of less than 24 hours. Assuming an even distribution of coexposure events lasting less than 24 hours among patients, this would result in 3.4 exposure days per patient over the course of 6 months. Consistently, no patients from the HAVEN 3 trial (or the HOHOEMI trial) developed new FVIII inhibitors. Compared with data from the INSIGHT study, inhibitors developed with a cumulative incidence of 5.3\% after a median of 28 exposure days. ${ }^{87}$ It is therefore conceivable that, even in case FVIII concentrates are needed to cope with breakthrough bleeding under treatment with emicizumab, exposure to FVIII is most often limited to less than 24 hours ( $=1$ exposure day), involves mostly low doses of FVIII, and thereby allows keeping inhibitor development to a minimum.

Further, emicizumab treatment in infants or small children would preclude constant intravenous injections, which presents a great burden of illness and leads to traumatization of young patients. Importantly, early treatment initiation in PUPs may be able to decrease the incidence of intracranial hemorrhage, a potentially fatal complication encountered in infants with impaired hemostasis. ${ }^{88}$

The efficacy of emicizumab prophylaxis has been successfully proven in infants and small children. ${ }^{89}$ However, uncertainties about the PK and clinical pharmacology of monoclonal antibodies in pediatric patients remain, with evidence suggesting age-associated PK/PD differences in addition to size-based differences. ${ }^{90}$

Third, an emicizumab-only treatment regimen may just as well be used in patients with inhibitors as prophylaxis with emicizumab has been shown to be superior to prophylaxis with BPAs. ABRs under emicizumab have become so low that treatment of breakthrough bleedings with BPAs (primarily rFVIIa) seems to be a more reasonable treatment option compared with undergoing inhibitor eradication with ITI.

\section{Emicizumab versus Immune Tolerance Induction}

Despite the unprecedented efficacy of emicizumab in patients with hemophilia A with or without inhibitors in reducing the rate of bleeding events, doubts about its unrestricted routine use have been expressed. ${ }^{91}$

First, hemophilic patients with inhibitors show both an increased risk of bleeding-associated death and a $70 \%$ increase in overall mortality, ${ }^{31}$ concluding that inhibitor eradication by ITI should remain the primary goal in this particular patient population. On the other hand, the efficacy of emicizumab is independent of presence of FVIII inhibitors, possibly rendering inhibitor eradication irrelevant. Further, even in the presence of inhibitors, emicizumab significantly reduced the $A B R$, which may also result in a reduction of mortality.
Second, as mentioned above, treatment of bleeding episodes in hemophilic patients with inhibitors receiving emicizumab is problematic and the procoagulatory potential of concomitant emicizumab and APCC or even rFVIIa yet remains insufficiently studied. ${ }^{92}$ However, due to the inferiority of BPAs to $\mathrm{FVIII}^{38-40}$ with regard to treatment of bleeding episodes and possibly serious adverse events when using APCC in a patient receiving emicizumab, the use of APCC should be avoided as much as possible, which is only feasible when patients undergo inhibitor eradication with ITI. ${ }^{91,93}$

Another argument in favor of ITI rather than treatment with emicizumab involves a possible future treatment method of hemophilia A-gene therapy. This is discussed below.

Last, the lack of sufficient long-term data regarding efficacy, safety, and immunogenicity precludes the universal application of emicizumab but, on the other hand, warrants generation of more data which will help inform health care treatment decisions.

There are multiple treatment regimens for ITI consisting of high and low FVIII dosing regimens. Patients undergoing high-dose ITI have fewer bleeding events during treatment and achieve tolerance faster ${ }^{41,94}$; however, high-dose ITI implies an intense treatment schedule and is very expensive. Patients undergoing low-dose ITI bleed more but treatment is less burdensome and more cost-effective. ${ }^{41}$

Prophylactic use of emicizumab may be used in patients undergoing ITI as supportive treatment to minimize bleeding events. This has already been successfully tested ${ }^{95}$ and is considered a potential new approach for the management of inhibitor patients. ${ }^{96}$

Subsequently, instead of continued FVIII replacement, patients could be switched to emicizumab-only treatment; however, the question of whether completed ITI necessitates the continued administration of FVIII to maintain tolerance remains unanswered. ${ }^{91}$

Given the quite recent introduction of emicizumab, its use is not included in the 2012 guidelines for the management of hemophilia. ${ }^{2}$ Emicizumab's place in succeeding guidelines will be of interest, given the presence of widely different treatment approaches.

\section{Q4W Emicizumab Dosing Regimen}

Of the three currently approved dosing intervals, Q4W was associated with higher ABRs of treated bleeding rates (HAVEN 2: 2.2 and HAVEN 4: 2.4). Consistently, increased ABRs were combined with lower PK results (Q4W mean trough level: $38-40 \mu \mathrm{g} / \mathrm{mL}$ ), which were, however, expected based on PK simulations ${ }^{97}$ and associated with efficacy, similar to those of the other tested dosing intervals. The larger peak-trough fluctuation combined with moderate to high interindividual variability in emicizumab exposure leading to suboptimal emicizumab plasma concentrations may account for increased ABRs of treated bleeds as found in HAVEN 2 and 4. The decision to choose the Q4W dosing regimen, which appeals to both patients and physicians, must be carefully weighed against its potential ABR increase compared with the $\mathrm{QW}$ and $\mathrm{Q} 2 \mathrm{~W}$ regimens and is therefore ultimately chosen based on individual patient preferences. 


\section{Emicizumab in Acquired Hemophilia A}

While emicizumab has not been approved by health authorities for the treatment of acquired hemophilia $A$, there are published reports of benefit from treatment with emicizumab. ${ }^{98-100}$ So far, over 15 male and female patients with acquired hemophilia $A$ have been reported to be treated with emicizumab, all of which had excellent response to treatment. ${ }^{101,102}$ The use of emicizumab in this patient population may also offer the option to scale down on the immunosuppressive treatment with corticosteroids to avoid side effects in a typically frail elderly population, and to save the enormous costs of bypassing therapy. A clinical trial with emicizumab and reduced immunosuppression is currently under development (NCT04188639) and is scheduled to start in mid-2020.

\section{Emicizumab in von Willebrand Disease}

The bleeding phenotype for the expected persistent complete absence of von Willebrand Disease, as in type 3 von Willebrand disease, together with moderate FVIII-like activity provided by emicizumab remains to be demonstrated. ${ }^{103}$

\section{Economic Considerations}

Given the lifelong persistence of hemophilia A, the use of emicizumab greatly depends on its costs. In a cost-effectiveness analysis, emicizumab prophylaxis was associated with fewer bleeding events and increased quality of life at a lower total cost compared with prophylaxis using BPAs and no prophylaxis. ${ }^{104}$ A study involving a model-based prediction of clinical and economic outcomes confirmed emicizumab's lower financial impact while at the same time improving patient outcomes. ${ }^{105} \mathrm{~A}$ recent cost-effectiveness analysis, comprising a Markov model and a budget impact model, further substantiates the findings of previous studies. ${ }^{106}$

\section{Novel Therapeutic Options to Treat Hemophilia}

Several other novel treatment strategies to combat hemophilia are currently under investigation. ${ }^{107}$

One approach to restoring hemostatic balance is by targeting the natural anticoagulant TF pathway inhibitor (TFPI). TFPI is part of the endogenous anticoagulation system and suppresses early stages of coagulation by inhibiting TF-VIIa (-Fig. 1A) and early forms of prothrombinase. ${ }^{108}$ Inhibition of TFPI could lead to increasing procoagulant activity thereby counteracting the anticoagulative nature of hemophilia and achieving hemostatic balance. A phase 1 trial of concizumab, a monoclonal antibody directed against TFPI, showed a favorable safety profile and increased d-dimer levels and prothrombin $1+2$ fragments in a dose-dependent manner. ${ }^{109}$ Phase 2 and 3 trials are currently ongoing.

Another way to induce a more procoagulant state is interference with antithrombin, a central component of the coagulation system. Fitusiran, a novel RNA interference therapy, was able to lower antithrombin levels and increase thrombin generation in patients with hemophilia A or B in a phase 1 dose-escalation study. ${ }^{110}$

A third promising treatment approach for hemophilia $A$ is gene therapy. In a phase $1 / 2$, dose-escalation, safety, tolerability, and efficacy study, nine men with hemophilia A without inhibitors received a single intravenous infusion of a codon-optimized adeno-associated virus serotype 5 (AAV5) vector encoding a B-domain-deleted human FVIII (AAV5-hFVIII-SQ). ${ }^{111}$ The study yielded promising results as patients receiving the high dose $\left(6 \times 10^{13}\right.$ vector genomes, $n=7$ ) showed FVIII activity of more than $5 \mathrm{IU} / \mathrm{dL}$ between weeks 2 and 9, with six of seven patients even reaching normal FVIII activity of $>50 \mathrm{IU} / \mathrm{dL}$ that were maintained at 1 year after infusion. This potential treatment option is only viable provided no FVIII inhibitors are present, further alluding to a benefit of continued indication for ITI treatment.

\section{Conclusion}

Emicizumab is a novel, bispecific antibody that restores the hemostatic balance in patients with hemophilia A irrespective of the presence of inhibitors. Across all three dosage regimens, it impressively reduced the ABR of the majority of patients to zero in several clinical trials. Emicizumab showed a good safety and tolerability profile, but concurrent use of emicizumab and APCC should be avoided due to increased risk of TMA and thromboembolic events. Use of emicizumab may be extended to patients with acquired hemophilia $A$ in the future. While additional long-term follow-up data are accumulated, emicizumab is set to revolutionize the treatment of hemophilia A as it unifies both efficacy and practicality compared with other currently available treatment options.

Funding

G.G. is supported by grant SFB54-P04 from the Austrian Science Funds.

\section{Conflict of Interest}

B.J. reports grants and personal fees from Roche, during the conduct of the study. P.K. reports personal fees from Roche, during the conduct of the study; grants, personal fees, and nonfinancial support from Baxalta/Shire/ Takeda; grants, personal fees, and nonfinancial support from Novo Nordisk; grants, personal fees, and nonfinancial support from CSL Behring; grants, personal fees, and nonfinancial support from Ablynx/Sanofi; and grants from Biotest, outside the submitted work.

\section{References}

1 Soucie JM, Evatt B, Jackson D; The Hemophilia Surveillance System Project Investigators. Occurrence of hemophilia in the United States. Am J Hematol 1998;59(04):288-294

2 Srivastava A, Brewer AK, Mauser-Bunschoten EP, et al; Treatment Guidelines Working Group on Behalf of The World Federation Of Hemophilia. Guidelines for the management of hemophilia. Haemophilia 2013;19(01):e1-e47

3 Mannucci PM, Tuddenham EG. The hemophilias-from royal genes to gene therapy. N Engl J Med 2001;344(23):1773-1779

4 Blanchette VS, Key NS, Ljung LR, Manco-Johnson MJ, van den Berg HM, Srivastava A; Subcommittee on Factor VIII, Factor IX and Rare Coagulation Disorders of the Scientific and Standardization Committee of the International Society on Thrombosis 
and Hemostasis. Definitions in hemophilia: communication from the SSC of the ISTH. J Thromb Haemost 2014;12(11): 1935-1939

5 Franchini M, Favaloro EJ, Lippi G. Mild hemophilia A. J Thromb Haemost 2010;8(03):421-432

6 Carcao MD. The diagnosis and management of congenital hemophilia. Semin Thromb Hemost 2012;38(07):727-734

7 Weyand AC, Pipe SW. New therapies for hemophilia. Blood 2019; 133(05):389-398

8 Franchini M, Mannucci PM. Hemophilia A in the third millennium. Blood Rev 2013;27(04):179-184

9 Kuijlaars IAR, Timmer MA, de Kleijn P, Pisters MF, Fischer K. Monitoring joint health in haemophilia: Factors associated with deterioration. Haemophilia 2017;23(06):934-940

10 Jansen NW, Roosendaal G, Lafeber FP. Understanding haemophilic arthropathy: an exploration of current open issues. $\mathrm{Br} \mathrm{J}$ Haematol 2008;143(05):632-640

11 Wheeler AP, Gailani D. The intrinsic pathway of coagulation as a target for antithrombotic therapy. Hematol Oncol Clin North Am 2016;30(05):1099-1114

12 Baker CJ, Smith SA, Morrissey JH. Polyphosphate in thrombosis, hemostasis, and inflammation. Res Pract Thromb Haemost 2018; 3(01):18-25

13 Lenting PJ, van Mourik JA, Mertens K. The life cycle of coagulation factor VIII in view of its structure and function. Blood 1998;92 (11):3983-3996

14 Kamikubo Y, Mendolicchio GL, Zampolli A, et al. Selective factor VIII activation by the tissue factor-factor VIIa-factor Xa complex. Blood 2017;130(14):1661-1670

15 Gale AJ. Continuing education course \#2: current understanding of hemostasis. Toxicol Pathol 2011;39(01):273-280

16 Aledort L, Mannucci PM, Schramm W, Tarantino M. Factor VIII replacement is still the standard of care in haemophilia A. Blood Transfus 2019;17(06):479-486

17 Mannucci PM. Benefits and limitations of extended plasma halflife factor VIII products in hemophilia A. Expert Opin Investig Drugs 2020;29(03):303-309

18 Oldenburg J. Optimal treatment strategies for hemophilia: achievements and limitations of current prophylactic regimens. Blood 2015;125(13):2038-2044

19 Fischer K, van der Bom JG, Molho P, et al. Prophylactic versus ondemand treatment strategies for severe haemophilia: a comparison of costs and long-term outcome. Haemophilia 2002;8(06): 745-752

20 Gringeri A, Lundin B, von Mackensen S, Mantovani L, Mannucci PM; ESPRIT Study Group. A randomized clinical trial of prophylaxis in children with hemophilia A (the ESPRIT Study). J Thromb Haemost 2011;9(04):700-710

21 Manco-Johnson MJ, Abshire TC, Shapiro AD, et al. Prophylaxis versus episodic treatment to prevent joint disease in boys with severe hemophilia. N Engl J Med 2007;357(06): 535-544

22 Manco-Johnson MJ, Soucie JM, Gill JC; Joint Outcomes Committee of the Universal Data Collection, US Hemophilia Treatment Center Network. Prophylaxis usage, bleeding rates, and joint outcomes of hemophilia, 1999 to 2010: a surveillance project. Blood 2017;129(17):2368-2374

23 Aledort LM, Haschmeyer RH, Pettersson H; The Orthopaedic Outcome Study Group. A longitudinal study of orthopaedic outcomes for severe factor-VIII-deficient haemophiliacs. J Intern Med 1994;236(04):391-399

24 Santagostino E, Young G, Carcao M, Mannucci PM, Halimeh S, Austin S. A contemporary look at FVIII inhibitor development: still a great influence on the evolution of hemophilia therapies. Expert Rev Hematol 2018;11(02):87-97

25 Collins PW, Fischer K, Morfini M, Blanchette VS, Björkman S; International Prophylaxis Study Group Pharmacokinetics Expert Working Group. Implications of coagulation factor VIII and IX pharmacokinetics in the prophylactic treatment of haemophilia. Haemophilia 2011;17(01):2-10

26 Troisi CL, Hollinger FB, Hoots WK, et al. A multicenter study of viral hepatitis in a United States hemophilic population. Blood 1993;81(02):412-418

27 Kumar A, Kulkarni R, Murray DL, et al. Serologic markers of viral hepatitis A, B, C, and D in patients with hemophilia. J Med Virol 1993;41(03):205-209

28 Kroner BL, Rosenberg PS, Aledort LM, Alvord WG, Goedert JJ. HIV-1 infection incidence among persons with hemophilia in the United States and western Europe, 1978-1990. Multicenter Hemophilia Cohort Study. J Acquir Immune Defic Syndr (1988) 1994;7(03):279-286

29 Soucie JM, Richardson LC, Evatt BL, et al; Hemophilia Surveillance System Project Investigators. Risk factors for infection with $\mathrm{HBV}$ and $\mathrm{HCV}$ in a largecohort of hemophiliac males. Transfusion 2001;41(03):338-343

30 Arnold DM, Julian JA, Walker IR; Association of Hemophilia Clinic Directors of Canada. Mortality rates and causes of death among all HIV-positive individuals with hemophilia in Canada over 21 years of follow-up. Blood 2006;108(02):460-464

31 Walsh CE, Soucie JM, Miller $\mathrm{CH}$; United States Hemophilia Treatment Center Network. Impact of inhibitors on hemophilia A mortality in the United States. Am J Hematol 2015;90(05): 400-405

32 Monahan PE, Baker JR, Riske B, Soucie JM. Physical functioning in boys with hemophilia in the U.S. Am J Prev Med 2011;41(06, Suppl 4):S360-S368

33 Morfini M. Articular status of haemophilia patients with inhibitors. Haemophilia 2008;14(Suppl 6):20-22

34 Brown TM, Lee WC, Joshi AV, Pashos CL. Health-related quality of life and productivity impact in haemophilia patients with inhibitors. Haemophilia 2009;15(04):911-917

35 Guh S, Grosse SD, McAlister S, Kessler CM, Soucie JM. Healthcare expenditures for males with haemophilia and employer-sponsored insurance in the United States, 2008. Haemophilia 2012;18 (02):268-275

36 Ullman M, Hoots WK. Assessing the costs for clinical care of patients with high-responding factor VIII and IX inhibitors. Haemophilia 2006;12(Suppl 6):74-79

37 Kulkarni R, Aledort LM, Berntorp E, et al. Therapeutic choices for patients with hemophilia and high-titer inhibitors. Am J Hematol 2001;67(04):240-246

38 Witmer C, Young G. Factor VIII inhibitors in hemophilia A: rationale and latest evidence. Ther Adv Hematol 2013;4(01): 59-72

39 Leissinger CA. Prevention of bleeds in hemophilia patients with inhibitors: emerging data and clinical direction. Am J Hematol 2004;77(02):187-193

40 Dimichele D, Rivard G, Hay C, Antunes S. Inhibitors in haemophilia: clinical aspects. Haemophilia 2004;10(Suppl 4):140-145

41 Hay CR, DiMichele DM; International Immune Tolerance Study. The principal results of the International Immune Tolerance Study: a randomized dose comparison. Blood 2012;119(06): 1335-1344

42 Mariani G, Siragusa S, Kroner BL. Immune tolerance induction in hemophilia A: a review. Semin Thromb Hemost 2003;29(01): 69-76

43 Waters B, Lillicrap D. The molecular mechanisms of immunomodulation and tolerance induction to factor VIII. J Thromb Haemost 2009;7(09):1446-1456

44 Gringeri A, Mantovani LG, Scalone L, Mannucci PM; COCIS Study Group. Cost of care and quality of life for patients with hemophilia complicated by inhibitors: the COCIS Study Group. Blood 2003;102(07):2358-2363

45 Cafuir LA, Kempton CL. Current and emerging factor VIII replacement products for hemophilia A. Ther Adv Hematol 2017;8(10): 303-313 
46 European Medicines Agency. Assessment report: Hemlibra. January 25, 2018. Available at: https://www.ema.europa.eu/en/documents/assessment-report/hemlibra-epar-public-assessment-report_en.pdf. Accessed June 26, 2020

47 Kitazawa T, Igawa T, Sampei Z, et al. A bispecific antibody to factors IXa and $X$ restores factor VIII hemostatic activity in a hemophilia A model. Nat Med 2012;18(10):1570-1574

48 Kitazawa T, Esaki K, Tachibana T, et al. Factor VIIIa-mimetic cofactor activity of a bispecific antibody to factors IX/IXa and $\mathrm{X} / \mathrm{Xa}$, emicizumab, depends on its ability to bridge the antigens. Thromb Haemost 2017;117(07):1348-1357

49 Lenting PJ, Denis CV, Christophe OD. Emicizumab, a bispecific antibody recognizing coagulation factors IX and X: how does it actually compare to factor VIII? Blood 2017;130(23): 2463-2468

50 Blair HA. Emicizumab: a review in haemophilia A. Drugs 2019;79 (15):1697-1707

51 Uchida N, Sambe T, Yoneyama K, et al. A first-in-human phase 1 study of ACE910, a novel factor VIII-mimetic bispecific antibody, in healthy subjects. Blood 2016;127(13):1633-1641

52 Shima M, Hanabusa H, Taki M, et al. Factor VIII-mimetic function of humanized bispecific antibody in hemophilia A. N Engl J Med 2016;374(21):2044-2053

53 Pipe SW, Shima M, Lehle M, et al. Efficacy, safety, and pharmacokinetics of emicizumab prophylaxis given every 4 weeks in people with haemophilia A (HAVEN 4): a multicentre, openlabel, non-randomised phase 3 study. Lancet Haematol 2019;6 (06):e295-e305

54 F. Hoffmann-La Roche Ltd. Investigator's Brochure: Emicizumab. November 2017

55 Mahlangu J, Oldenburg J, Paz-Priel I, et al. Emicizumab prophylaxis in patients who have hemophilia a without inhibitors. $\mathrm{N}$ Engl J Med 2018;379(09):811-822

56 Oldenburg J, Mahlangu JN, Kim B, et al. Emicizumab prophylaxis in hemophilia A with inhibitors. N Engl J Med 2017;377(09):809-818

57 Young G, Liesner R, Chang T, et al. A multicenter, open-label phase 3 study of emicizumab prophylaxis in children with hemophilia A with inhibitors. Blood 2019;134(24):2127-2138

58 Genentech Inc. Hemlibra ${ }^{\circledR}$ (emicizumab-kxwh) injection, for subcutaneous use: US prescribing information. Available at: http://dailymed.nlm.nih.gov/dailymed/drugInfo.cfm? setid=2483adba-fab6-4d1b-96c5-c195577ed071. Published 2018. Accessed October 14, 2019

59 Callaghan M, Negrier C, Paz-Priel I, et al. Emicizumab treatment is efficacious and well tolerated long term in persons with haemophilia A (PwHA) with or without FVIII inhibitors: pooled data from four HAVEN studies. Paper presented at: ISTH Congress 2019, Melbourne, Australia; July 6-10, 2019

60 Shima M, Nogami K, Nagami S, et al. A multicentre, open-label study of emicizumab given every 2 or 4 weeks in children with severe haemophilia A without inhibitors. Haemophilia 2019;25 (06):979-987

61 Jiménez-Yuste V, Klamroth R, Castaman G, et al. A single-arm, multicentre, open-label, phase III clinical trial to evaluate the safety and tolerability of prophylactic emicizumab in persons with haemophilia A (PwHA) with FVIII inhibitors (STASEY): interim analysis results. Paper presented at: ISTH Congress 2019, Melbourne, Australia; July 6-10, 2019

62 Ebbert PT, Xavier F, Seaman CD, Ragni MV. Emicizumab prophylaxis in patients with haemophilia A with and without inhibitors. Haemophilia 2020;26(01):41-46

63 Santagostino E, Oldenburg J, Chang T, et al. Surgical experience from four phase III studies (HAVEN 1-4) of emicizumab in persons with haemophilia A (PwHA) with or without FVIII inhibitors. Paper presented at: ISTH Congress 2019, Melbourne, Australia; July 6-10, 2019

64 Seaman CD, Ragni MV. Emicizumab use in major orthopedic surgery. Blood Adv 2019;3(11):1722-1724
65 Biron-Andreani CD-CI, Navarro R, Garcia-Gournay C, Theron A, Santagostino E, Schved J-F. Management of surgery in hemophilia A patients with inhibitors during emicizumab prophylaxis. Paper presented at: ISTH Congress 2019, Melbourne, Australia; July 6-10, 2019

66 Dane KE, Lindsley JP, Streiff MB, Moliterno AR, Khalid MK, Shanbhag S. Successful use of emicizumab in a patient with refractory acquired hemophilia A and acute coronary syndrome requiring percutaneous coronary intervention. Res Pract Thromb Haemost 2019;3(03):420-423

67 Oldenburg J, Mahlangu JN, Bujan W, et al. The effect of emicizumab prophylaxis on health-related outcomes in persons with haemophilia A with inhibitors: HAVEN 1 Study. Haemophilia 2019;25(01):33-44

68 Müller J, Pekrul I, Pötzsch B, Berning B, Oldenburg J, Spannagl M. Laboratory monitoring in emicizumab-treated persons with hemophilia A. Thromb Haemost 2019;119(09):1384-1393

69 Adamkewicz JI, Chen DC, Paz-Priel I. Effects and interferences of emicizumab, a humanised bispecific antibody mimicking activated factor VIII cofactor function, on coagulation assays. Thromb Haemost 2019;119(07):1084-1093

70 Calatzis AKN, Levy G, Adamkewicz J. Effect of emicizumab - a humanized bispecific antibody mimicking FVIII cofactor function - on a variety of assay systems. Paper presented at: 2016 European Congress on Thrombosis and Haemostasis, Hague, Netherlands; 28-30 September, 2016

71 Calhoon W, McInerney M, Calatzis A, Chen DC, Adamkewicz J, Morris M. Evaluation of a dedicated calibrator and controls for emicizumab quantification. Paper presented at: THSNA 2018, San Diego, California, United States; March 8-10, 2018

72 Jenkins PV, Bowyer A, Burgess C, et al. Laboratory coagulation tests and emicizumab treatment A United Kingdom Haemophilia Centre Doctors' Organisation guideline. Haemophilia 2020;26 (01):151-155

73 Adamkewicz J, Soeda T, Kotani N, Calatzis A, Levy G. Effect of emicizumab (ACE910) - a humanized bispecific antibody mimicking FVIIIa cofactor function - on coagulation assays commonly in use for monitoring of hemophilia A patients. Paper presented at: 2017 Scientific Symposium of the Hemostasis and Thrombosis Research Society (HTRS), April 6-8, 2017; Scottsdale, AZ, United States

74 Yada K, Nogami K, Kasai R, Shima M. A novel hemostatic monitoring system convertible to FVIII activity based on nonactivated ROTEM (NATEM) for hemophilia A patients during emicizumab prophylaxis. 2018;132(Suppl 1):3782

75 Hartmann R, Feenstra T, Valentino L, Dockal M, Scheiflinger F. In vitro studies show synergistic effects of a procoagulant bispecific antibody and bypassing agents. J Thromb Haemost 2018

76 Nogami K, MatsumotoT, Tabuchi Y, et al. Modified clot waveform analysis to measure plasma coagulation potential in the presence of the anti-factor IXa/factor X bispecific antibody emicizumab. J Thromb Haemost 2018;16(06):1078-1088

77 Schmitt C, Adamkewicz JI, Xu J, et al. Pharmacokinetics (PK), pharmacodynamics (PD), and PK/PD relationships of emicizumab in persons with hemophilia A (PwHA) with inhibitors from adolescent/adult (HAVEN 1) and paediatric (HAVEN 2) phase 3 studies. Paper presented at: 11th Annual Congress of the European Association for Haemophilia and Allied Disorders (EAHAD), February 7-9, 2018 ; Madrid, Spain

78 Dargaud Y, Lienhart A, Janbain M, Le Quellec S, Enjolras N, Negrier $C$. Use of thrombin generation assay to personalize treatment of breakthrough bleeds in a patient with hemophilia and inhibitors receiving prophylaxis with emicizumab. Haematologica 2018;103(04):e181-e183

79 Levy GG, Asikanius E, Kuebler P, Benchikh El Fegoun S, Esbjerg S, Seremetis S. Safety analysis of rFVIIa with emicizumab dosing in congenital hemophilia A with inhibitors: experience from the HAVEN clinical program. J Thromb Haemost 2019;17(09):1470-1477 
80 Collins PW, Liesner R, Makris M, et al. Treatment of bleeding episodes in haemophilia A complicated by a factor VIII inhibitor in patients receiving Emicizumab. Interim guidance from UKHCDO Inhibitor Working Party and Executive Committee. Haemophilia 2018;24(03):344-347

81 Susen S, Gruel Y, Godier A, et al. Management of bleeding and invasive procedures in haemophilia A patients with inhibitor treated with emicizumab (Hemlibra ${ }^{\circledR}$ ): proposals from the French network on inherited bleeding disorders (MHEMO), the French Reference Centre on Haemophilia, in collaboration with the French Working Group on Perioperative Haemostasis (GIHP). Haemophilia 2019;25(05):731-737

82 Castaman G, Santoro C, Coppola A, et al. ad hoc Working Group. Emergency management in patients with haemophilia $A$ and inhibitors on prophylaxis with emicizumab: AICE practical guidance in collaboration with SIBioC, SIMEU, SIMEUP, SIPMeL and SISET. Blood Transfus 2020;18(02):143-151

83 Makris M, Iorio A, Lenting PJ. Emicizumab and thrombosis: The story so far. J Thromb Haemost 2019;17(08):1269-1272

$84 \mathrm{~W}$ Keith Hoots ADS. Treatment of bleeding and perioperative management in hemophilia A and B. Available at: https://www. uptodate.com/contents/treatment-of-bleeding-and-perioperative-management-in-hemophilia-a-and-b?search=hemophilia \&source=search_result\&selectedTitle=3 150\&usage_type=default\&display_rank=3\#H2996978111. Published 2020. Accessed February 13, 2020

85 Hermans C, Astermark J, De Moerloose P. Exposure to factor VIII and prediction of inhibitor development: exposure days vs. danger days, or both? J Thromb Haemost 2012;10(10): 2194-2196

86 Benson G, Auerswald G, Dolan G, et al. Diagnosis and care of patients with mild haemophilia: practical recommendations for clinical management. Blood Transfus 2018;16(06):535-544

87 Eckhardt CL, van Velzen AS, Peters M, et al; INSIGHT Study Group. Factor VIII gene (F8) mutation and risk of inhibitor development in nonsevere hemophilia A. Blood 2013;122(11):1954-1962

88 Kenet G, Barg AA, Nowak-Göttl U. Hemostasis in the very young. Semin Thromb Hemost 2018;44(07):617-623

89 Barg AA, Avishai E, Budnik I, et al. Emicizumab prophylaxis among infants and toddlers with severe hemophilia $A$ and inhibitors-a single-center cohort. Pediatr Blood Cancer 2019; 66(11):e27886

90 Temrikar ZH, Suryawanshi S, Meibohm B. Pharmacokinetics and clinical pharmacology of monoclonal antibodies in pediatric patients. Paediatr Drugs 2020;22(02):199-216

91 Young G. Implementing emicizumab in hemophilia inhibitor management: emicizumab should be prescribed after tolerance. Blood Adv 2018;2(20):2780-2782

92 Aledort LM. Deaths associated with emicizumab in patients with hemophilia A. N Engl J Med 2019;381(19):1878-1879

93 Ljung R, Auerswald G, Benson G, et al. Inhibitors in haemophilia A and $\mathrm{B}$ : management of bleeds, inhibitor eradication and strategies for difficult-to-treat patients. Eur J Haematol 2019;102(02): $111-122$

94 Ettingshausen CE, Kreuz W. The immune tolerance induction (ITI) dose debate: does the International ITI Study provide a clearer picture? Haemophilia 2013;19(Suppl 1):12-17
95 Batsuli G, Zimowski KL, Tickle K, Meeks SL, Sidonio RF Jr. Immune tolerance induction in paediatric patients with haemophilia $\mathrm{A}$ and inhibitors receiving emicizumab prophylaxis. Haemophilia 2019;25(05):789-796

96 Carcao M, Escuriola-Ettingshausen C, Santagostino E, et al; Future of Immunotolerance Treatment Group. The changing face of immune tolerance induction in haemophilia A with the advent of emicizumab. Haemophilia 2019;25(04):676-684

97 Yoneyama K, Schmitt C, Kotani N, et al. A pharmacometric approach to substitute for a conventional dose-finding study in rare diseases: example of phase III dose selection for emicizumab in hemophilia A. Clin Pharmacokinet 2018;57(09): 1123-1134

98 Al-Banaa K, Alhillan A, Hawa F, et al. Emicizumab use in treatment of acquired hemophilia A: a case report. Am J Case Rep 2019;20:1046-1048

99 Möhnle P, Pekrul I, Spannagl M, Sturm A, Singh D, Dechant C. Emicizumab in the treatment of acquired haemophilia: a case report. Transfus Med Hemother 2019;46(02):121-123

100 Knöbl P. Prevention and management of bleeding episodes in patients with acquired hemophilia A. Drugs 2018;78(18): 1861-1872

101 Knoebl P, Sperr W, Schellongowski P, et al. Emicizumab for the treatment of acquired hemophilia_A: lessons learned from 4 very different cases. Blood 2018;132(Suppl 1):2476

102 Knoebl P, Thaler J, Jilma-Stohlawetz P, Quehenberger P, Gleixner $\mathrm{K}$, Sperr WR. Emicizumab for the treatment of acquired hemophilia A. Blood 2020. Doi: 10.1182/blood.2020006315

103 Weyand AC, Flood VH, Shavit JA, Pipe SW. Efficacy of emicizumab in a pediatric patient with type 3 von Willebrand disease and alloantibodies. Blood Adv 2019;3(18):2748-2750

104 ICER. Emicizumab for hemophilia A with inhibitors: effectiveness and value. April 16, 2018. Available at: https://icer-review.org/wp-content/uploads/2017/08/ICER_Hemophilia_Final_Evidence_Report_041618.pdf. Accessed June 26, 2020

105 Mahajerin A, Zhou Z-Y, Raimundo K, et al. Model of short and long-term outcomes of emicizumab prophylaxis treatment for persons with hemophilia A. Blood 2018;132(Suppl 1):3511

106 Cortesi PA, Castaman G, Trifirò G, et al. Cost-effectiveness and budget impact of emicizumab prophylaxis in haemophilia $\mathrm{A}$ patients with inhibitors. Thromb Haemost 2020;120(02): 216-228

107 Spadarella G, Di Minno A, Milan G, et al. Paradigm shift for the treatment of hereditary haemophilia: towards precision medicine. Blood Rev 2020;39:100618

108 Peterson JA, Maroney SA, Mast AE. Targeting TFPI for hemophilia treatment. Thromb Res 2016;141(Suppl 2):S28-S30

109 Chowdary P, Lethagen S, Friedrich U, et al. Safety and pharmacokinetics of anti-TFPI antibody (concizumab) in healthy volunteers and patients with hemophilia: a randomized first human dose trial. J Thromb Haemost 2015;13(05):743-754

110 Pasi KJ, Rangarajan S, Georgiev P, et al. Targeting of antithrombin in hemophilia A or B with RNAi therapy. N Engl J Med 2017;377 (09):819-828

111 Rangarajan S, Walsh L, Lester W, et al. AAV5-factor VIII gene transfer in severe hemophilia A. N Engl J Med 2017;377(26): 2519-2530 